\begin{tabular}{|c|l|}
\hline Title & $\begin{array}{l}\text { Postembryonic development of sexually dimorphic glomeruli and related interneurons in the cockroach Periplaneta } \\
\text { americana }\end{array}$ \\
\hline Author(s) & Nishino, Hiroshi; Y oritsune, A tsushi; Mizunami, Makoto \\
\hline Citation & $\begin{array}{l}\text { Neuroscience Letters, 469(1), 60-64 } \\
\text { https://doi.org/10.1016/.neulet.2009.11.044 }\end{array}$ \\
\hline Issue Date & 2010-01-18 \\
\hline Doc URL & http://hdl.handle.net/2115/42702 \\
\hline Type & article (author version) \\
\hline File Information & NL469-1_60-64.pdf \\
\hline
\end{tabular}

Instructions for use 


\title{
Postembryonic development of sexually dimorphic glomeruli and related interneurons in the cockroach Periplaneta americana
}

\author{
Hiroshi Nishino $^{1 *}$, Atsushi Yoritsune ${ }^{1}$, Makoto Mizunami ${ }^{2}$ \\ ${ }^{1}$ Research Institute for Electronic Science, Hokkaido University, Sapporo 060-0812, \\ Japan \\ ${ }^{2}$ Faculty of Advanced Life Science, Hokkaido University, Sapporo 060-0810, Japan
}

The number of text pages:

The corresponding author: Hiroshi Nishino, Research Institute for Electronic Science, Hokkaido University, Sapporo 060-0812, Japan.

Tel: +81-11-706-2866

Fax:+81-11-706-4971

Email: nishino@es.hokudai.ac.jp

Abbreviations: AL: antennal lobe; EMG: electroantennogram; IACT: inner antenno-cerebral tract; MGC: macroglomerular complex; PNs: projection neurons 


\section{Abstract}

In most insects, sex pheromone is processed by an enlarged glomerular complex (macroglomerular complex, MGC) in the male antennal lobe (first-order olfactory center). The MGC of the American cockroach consists of two closely located A- and B-glomeruli which are responsible for processing the major sex pheromone components, periplanone-A and -B, respectively. Using anterograde dye injection, we investigated sexual dimorphism in sensory afferents and interneuron. The A- and B-glomeruli exist in the first larval instar of both sexes. The female MGC-homolog grows at a relatively constant rate (1.2-1.8 fold growth per molt) throughout development, whereas the male MGC shows a period of accelerated growth between the 5th and 9th instars, where volume can more than double in a single molt. These different growth patterns resulted in a 1:30 ratio in glomerular complex volumes of adult females versus males. In the female MGC-homolog, afferents originating from the dorsal and ventral antennal surfaces were biased toward anterior and posterior regions, and segregation of these afferents was less clear compared to the adult male. The staining of interneurons projecting to the protocerebrum revealed that projection patterns characteristic of sex pheromone processing appear in the late 8th instar in males, while possibly homologous projections in the female were far fewer in number. These results suggest that the glomerular complexes in pre-8th larval males, and probably females, are not differentiated for specific detection of sex pheromone. Male-specific projections for sex pheromone detection may be formed by modification of pre-existing neural circuitry.

Keywords: insects; olfactory afferents; macroglomerular complex; projection neurons: postembryonic development; sexual dimorphism

In most insects, chemical communication using sex pheromone is critical for reproductive success. The sex pheromone communication system involves the release of specific chemicals from a pheromone producer (emitter), the environmental transmission of these chemicals to a receiver, and the processing of these chemicals to mediate appropriate behavioral responses in the receiver [16]. In most insect species, the female is the pheromone producer and the male is the receiver [16]. Thus, males are equipped with an elaborate sensory system to detect low quantities of sex pheromones transmitted on long distances from females. This leads to "sexual dimorphism” in neural 
pathways for pheromone-processing. For example, the adult male antennae are characterized by a larger number of sensilla housing pheromone-receptive neurons than the adult female antennae $[20,21]$.

The axons of pheromone-receptive neurons, together with the axons of sensory neurons receptive to other volatile chemicals, form antennal nerves which project to the first-order olfactory center, called the antennal lobe (AL) $[4,6]$. The AL is functionally compartmentalized to process a limited spectrum of volatile chemicals [6,7]. Each compartment, called a glomerulus, offers a site where a large number of sensory neurons expressing cognate odorant receptors make synaptic connections with a smaller number of interneurons [6]. Sex pheromone in insects is usually a blend of structurally similar chemicals $[14,16]$, and these chemical compounds are processed by a specific set of glomeruli $[2,6]$.

In males, numerous pheromone-receptive afferents converge on a well-developed set of glomeruli, termed the macroglomerular complex (MGC). Detailed three-dimensional mapping of glomeruli in several insect species have revealed that the female ALs contain almost identical numbers of glomeruli to those of the male ALs, and often include a few glomeruli homologous to the male MGC. In the cockroach $B$. craniifer and fruit flies, the glomerulus in the female is much smaller $[8,17]$. In the sphinx moth and tobacco budworm moth, both male and female ALs possess a set of large glomeruli, but their organizational patterns differ [1,18].

The male cockroach $P$. americana possesses a MGC with two compartments, the A and B glomeruli [13], which are responsible for processing two major sex pheromone components, periplanone-A and periplanone-B, respectively [2,14]. Recently we showed that the MGC in the male is not formed by fusion of several glomeruli in late larval stages, as suggested in previous studies [15,22], but that the precursors of the Aand B-glomeruli already exist in the first larval instar [13]. We also showed differences in A- and B-glomeruli growth rates, both relative to each other and over time [13]. This raised the questions: does a female MGC-homolog exist? and if so, how are the homologous neural pathways organized in females? To obtain further insight into sexually dimorphic neural development, we investigated the postembryonic growth of female MGC-related glomeruli and their interneurons.

Larval instars and adult female and male cockroaches, $P$. americana with intact antennae reared in $12: 12 \mathrm{~h}$ light-dark cycle at $27^{\circ} \mathrm{C}$ were used. The procedures for 
discrimination of sex and larval stages, dissection of animals, dye-application and data analyses are identical to those used in our previous studies [12,13]. Briefly, single-dye labeling was used to measure the volume of afferents in the MGC, and double-dye labeling was used to observe topographic segregation of afferents originating from the two antennal surfaces. To stain axons of interneurons, a tapered glass electrode was filled with microemerald or microruby (Invitrogen, Eugene, Oregon, USA) and then attached to a microsyringe on a manipulator. The electrode was positioned into the center of the de-sheathed AL and dye was injected manually. The dye-injected specimens were left in a humid chamber at $4^{\circ} \mathrm{C}$ for $18-48 \mathrm{~h}$. Dye-injection to the AL was achieved in 2-3 animals for each developmental stage for each sex. Conventional intracellular staining in adult males was achieved as described previously [11].

In the cockroach, sensory neurons originating from sensilla on the dorsal surface of the antenna send axons to the dorsal nerve (magenta, Fig. 1a), while those on the ventral surface send axons to the ventral nerve (green, Fig. 1a). The two similarly sized antennal nerves grow thicker in later larval stages concurrent with addition of sensory axons originating from newly-emerged sensilla on the two antennal surfaces [21]. Accordingly, all glomeruli in the AL grow in parallel to the increasing number of incoming chemosensory afferents $[13,15]$.

As in other insects $[8,17,18]$, the adult male and female P. americana ALs contain almost identical numbers of glomeruli. A female MGC-homolog was located in the antero-lateral region of the AL (Fig. 1c), in the same location as the male MGC (Fig. 1b). The sizes of A and B glomeruli in females were much reduced compared to those in males, and were even smaller than more posterior glomeruli in the AL. The relative locations of the two glomeruli differed between sexes. The male A-glomerulus tended to be located antero-dorsally to the B-glomerulus (Fig. 1b) while the female A-glomerulus tended to be located antero-laterally to the B-glomerulus (Fig. 1c). The female morphology resembles the male MGC precursor at mid-larval stages [13].

As the number and location of glomeruli and related sensory tracts were very consistent from the first larval instar to the adult in both sexes [15], several landmarks used to identify the male MGC precursor [13] can be applied to the female homolog at different larval stages (Fig. 1c-i). The A and B glomeruli are the first glomeruli innervated by the single sensory tract diverging dorsally from the antennal nerve at the entrance of the AL (white arrowhead, Fig. 1b, c). The A- and B-glomeruli in females 
were consistently located: 1) postero-ventrally to the most antero-lateral glomerulus (g1, Fig. 1c,d); 2) antero-laterally to a sensory bundle (white arrow, Fig. 1d) projecting dorsally to innervate a fan-shaped glomerulus (g2, Fig. 1d); and 3) antero-medially to a glomerulus characterized by thick axon terminals (g3, Fig. 1d). The female A- and B-glomeruli already exist in the first larval instar (Fig. 1e) and grow in volume while retaining their relative locations in the adult (Fig. 1f-k).

The three-dimensional reconstructions of the adult female MGC homolog, in which afferents from the dorsal (magenta) and ventral (green) nerves were differentially labeled (Fig. 1j-q), revealed that sensory axons from the dorsal and ventral surfaces of the antennae are almost completely segregated anteriorly and posteriorly, respectively, before entering the glomeruli (black arrows, Fig. 1m,q). Reflecting this axonal organization, axon terminals tended to be similarly segregated in the A and B glomeruli (Fig. 1m,n,p,q, Supplementary data file A). The degree of segregation was smaller than in male late larval instars [13] or adult stages [12], and resembled more closely that in male mid-larval instars (Fig. 1i-q)[13]. The axon terminal segregation was not evident in other glomeruli including those innervated by the same sensory tract (e.g. see g1 in Supplementary data file A).

Plotting male and female A and B glomeruli volumes at different developmental stages (Fig. 2, $\mathrm{n}=2$ for each stage except $\mathrm{n}=1$ for 1 st larval instar) showed that growth patterns in both sexes were similar until about the fifth larval instar. The female MGC-homolog grew at a relatively constant rate (1.2-1.8x) until the 11th larval instar and then almost stopped growing. In contrast, male MGC precursor growth accelerated between the 5th and 9th instars (Fig. 2). This divergent growth resulted in a 1:30 ratio in adult female versus male glomerular complex volumes (Fig. 2, supplementary Table 1). Although there was some variability in the growth pattern of the female A-glomerulus, the data suggests the A- and B-glomeruli tended to grow at similar, relatively constant rates (Fig. 2). In contrast to males, in which MGC volume increased 430 fold from the first larval instar to the adult [13], the female MGC homolog increased only 17-fold. This is similar to the 14-fold volume increase seen in ordinary glomeruli from the first instar to the adult [15].

Next, we investigated sexual dimorphism in projection neurons (PNs). These relay olfactory signals processed in the $\mathrm{AL}$ to higher-order centers in the ipsilateral protocerebrum. The primary targets of PNs in the protocerebrum are calyces of the 
mushroom body and/or part of the lateral protocerebrum, also known as the lateral horn (Fig. 3a) [3,9]. The PNs were classified as either uniglomerular PNs which spread dendrites to a single glomerulus (Fig. 3f,g), or multiglomerular PNs which spread dendrites amongst multiple glomeruli [9].

There are about twenty uniglomerular PNs originating from the MGC in each hemisphere of the adult male brain [4], here collectively termed MGCPNs. These have dendrites in either the A- or B-glomerulus. Single staining showed that the axons of MGCPNs with dendrites in the B-glomerulus proceed via the inner antenno-cerebral tract (IACT, Fig. 3f). The axons send out collaterals to the calyces of the mushroom body and then project mainly to the antero-medial region of the lateral horn, with the remainder terminating in the posterior region of the lateral horn (indicated by white arrow, Fig. 3h). Axon terminals in the antero-medial region belonged exclusively to MGCPNs (Fig. 3b,d; Supplementary data file B) [4,9] in our samples and had a dense, fibrous appearance (Fig. 3h). However, we cannot exclude the possibility of axon terminals originating from other PNs, especially if they were few in number. The uniglomerular PNs having dendrites in ordinary glomeruli resembled MGCPNs in overall projection profiles (Fig. 3g), but their termination fields in the lateral horn were generally limited to the posterior region of the lateral horn, in which axon terminals were organized into discrete buttons (Fig. 3i) [11].

Morphologically the PNs were similar in both sexes at all developmental stages, as has been reported previously [15]. An important exception is the very few projections to the antero-medial region of the lateral horn in females (Fig. 3c,j). The axon terminals were sparse and appeared to be limited to the posterior side of the antero-medial region (Fig. 3c, Supplementary data file C). We could not confirm whether these terminals are originating from PNs via IACT or PNs via other tracts. (Fig. 3c,e,j; Supplementary data file C). In males, projections to the antero-medial region were few or absent until the mid 8th larval instars (Fig. 3k; Supplementary data files D), but became very apparent in the late 8th larval instar (Fig. 31). The triangular-shaped branching of newly-emerged axon terminals resembled that in the adult (Fig. 3l, Supplementary data file E), which suggests the projections to the antero-medial region mature rapidly.

The present study reports two novel findings. Firstly, the female MGC homolog in the American cockroach exists in the first larval instar and grows at a relatively constant rate (Figs. 1,2). Second, compared to males, females possess far fewer axon terminals in 
the neuropil which are presumably specialized for sex pheromone processing (Fig. 3).

Sexual dimorphism in the glomerular complex became evident from the sixth larval instar (Fig. 2). Prior to this the volume of the female MGC homolog was almost equal to that of the male MGC precursor (Fig. 2), and the relative positions of A- and B glomeruli and the organization patterns of afferents in adult females were similar to those of males at the fifth to sixth larval instars (Fig. 1). These results suggest that the adult female MGC homolog is equivalent to that of the male MGC precursor at mid-larval stages.

In the cockroach $B$. craniifer, the male macroglomerulus and its female homolog grow at similar, constant rates and have similar volumes until the last instar. At the imaginal ecdysis, the former increases in size much more than the latter [17]. This differs from the present findings in $P$. americana, indicating that the degree of sex-dependent developmental divergence between the male MGC and the female MGC homolog is species dependent.

Sex-dependent divergence in development is also seen in the antennae of $P$. americana. The male antennae are characterized by a metamorphic change from female-like sensilla to male-specific sensilla. In adult males, sensory neurons responding to either periplanone-A or B are co-housed in long, single-walled ( $s w B$ ) sensilla distributed along the antennae [21]. About 50\% of long sw B sensilla are derived from shorter type $s w B$ sensilla, present on the nymphal antenna, whereas the other 50\% are newly-formed at the imaginal ecdysis [21]. In contrast, adult females retain nymph-like shorter type $s w B$ sensilla [21]. The topical application of juvenile-hormone-mimic to the male antenna in late larval instars prevents both differentiation of shorter type sw B sensilla and the increase in number of male-specific sensilla [20] suggesting an inhibitory action of juvenile hormone to the formation of male-specific sensilla.

In contrast, sexual dimorphism in glomeruli of the moth $M$. sexta occurs during early pupal stages and precedes formation of ordinary glomeruli [19]. The female homolog of the "cumulus", one of the three glomeruli of the MGC, is well-developed in the female and is responsible for processing linalool, a volatile from a host-plant on which they prefer to lay their eggs [7]. Unlike holometabolous insects that change habitats and food preference greatly from larva to adult, cockroaches of different ages and sexes tend to occupy the same habitat [5]. Thus, female cockroaches appear not to require functional 
specialization in the MGC-homolog as in $M$. sexta. Electroantennogram (EAG) recordings from female cockroach antennae show sensitivity to periplanone-A and B from 7th instar to adult, although the EAG amplitudes are smaller than those in males [10]. Sensilla responsible for periplenone detection have not yet been determined in females. To study potential contributions of the female sw B sensilla on periplanone detection, electrophysiological recordings from female $s w B$ sensilla will be required.

The male MGCPNs were characterized by dense axonal arborizations in the antero-medial region of the lateral horn. We could only stain a few arborizations in the homologous regions in larval and adult females (Fig. 3). As the volume and postembryonic growth of the female MGC homolog are comparable to those of ordinary glomeruli, rather than those of the male MGC (Figs. 1, 2) [15], we estimate that 1 or 2 PNs may originate from the A or B glomerulus in females, similar to the number of PNs originating from an ordinary glomerulus [4].

The antero-medial region of the lateral horn did not exhibit distinct PN terminations in either sex up to the 8th instar (Fig. 3). Terminations were clear from the late 8th instar in males only (Fig. 3), suggesting that the male-specific pathways could be formed by modification of the pre-existing, female-like pathways, analogous to the development of sexually dimorphic antennal sensilla. As it is presumed that integration of sensory signals and their conversion to behavioral decisions occurs in the neural pathway involving the lateral horn [3] this morphologic change must be preparatory to initiation of pheromone-triggered orientation behavior in adult males to adult females [5].

Intriguingly, the formation of male-specific projections to the lateral horn occurs later than the male-specific glomerular volume increase which starts from the 6th instar (Fig. 2). We suspect that this delay allows development of the dynamic functional changes of interneuronal pathways required to process sex pheromone which is completed between the pre-8th instars and post-8th instars. No cell divisions of deutocerebral neurons have been observed during postembryonic development in $P$. americana [15], and the male-specific projections appeared to form relatively in short term (Fig. 3) which supports the view that these projections are derived from pre-existing PNs rather than by the addition of new PNs. As individual MGCPNs resemble ordinary uniglomerular PNs in their overall projection profiles [4,9], and as they partly share the same axonal termination fields (Fig. 3), this would provide favorable conditions for the metamorphic change of PNs by slight modification of axon 
terminals in calyces of the mushroom body and in the lateral horn [11]. The metamorphic change of PNs will offer an excellent model for investigating neural plasticity for future study.

Acknowledgments: We thank Dr. J. Hill University of Otago, Christchurch for helpful comments on the manuscript. This work was supported by ministry of Education, Science, Technology, Sports and Culture of Japan; Grant numbers: 20570066 to HN.

\section{References}

[1] B.G. Berg, C.G. Galizia, R. Brandt, H. Mustaparta, Digital atlases of the antennal lobe in two species of tobacco budworm moths, the oriental Helicoverpa assulta (male) and the American Heliothis virescens (male and female). J. Comp. Neurol. 446 (2002) 123-134.

[2] M. Burrows, J. Boeckh, J. Esslen, Physiological and morphological properties of interneurons in the deutocerebrum of male cockroaches which respond to female pheromone. J. Comp. Physiol. 145 (1982) 447-457.

[3] S.R. Datta, M.L. Vasconcelos, V. Ruta, S. Luo, A. Wong, E. Demir, J. Flores, K. Balonze, B.J. Dickson, R. Axel, The Drosophila pheromone cVA activates a sexually dimorphic neural circuit. Nature 452 (2008) 473-477.

[4] K.D. Ernst, J. Boeckh, A neuroanatomical study on the organization of the central antennal pathways in insects. III. Neuroanatomical characterization of physiologically defined response types of deutocerebral neurons in Periplaneta americana. Cell Tissue Res. 229 (1983) 1-22.

[5] C. Gemeno, C. Schal, Sex pheromones of cockroaches. In: Carde RT, Millar JG (Ed.) Advances in insect chemical ecology. Cambridge University Press, Cambridge, 2004, pp. 179-247.

[6] J.G. Hildebrand, Olfactory control of behavior in moths: central processing of odor 
information and the functional significance of olfactory glomeruli. J. Comp. Physiol. 178 (1996) 5-19.

[7] J.R. King, T.A. Christensen, J.G. Hildebrand, Response characteristics of an identified, sexually dimorphic olfactory glomerulus. J. Neurosci. 20 (2000) 2391-2399.

[8] Y. Kondoh, K.Y. Kaneshiro, K. Kimura, D. Yamamoto, Evolution of sexual dimorphism in the olfactory brain of Hawaiian Drosophila. Proc. R. Soc. Lond. B 270 (2003) 1005-1013.

[9] D. Malun, U. Waldow, D. Kraus, J. Boeckh, Connections between the deutocerebrum and the protocerebrum, and neuroanatomy of several classes of deutocerebral projection neurons in the brain of male Periplaneta americana. J. Comp. Neurol. 329 (1993) 143-162.

[10] C. Nishino, R. Kimura, Olfactory receptor responses of the nymphal American cockroach to sex pheromones and their mimics. Comp. Biochem. Physiol. (A) 72 (1982) 237-242.

[11] H. Nishino, S. Yamashita, Y. Yamazaki, M. Nishikawa, F. Yokohari, M. Mizunami, Projection neurons originating from thermo- and hygrosensory glomeruli in the antennal lobe of the cockroach. J. Comp. Neurol. 455 (2003) 40-55.

[12] H. Nishino, M. Mizunami, Sensilla position on antennae influences afferent terminal location in glomeruli. NeuroReport 18 (2007) 1765-1769.

[13] H. Nishino, A. Yoritsune, M. Mizunami, Different growth patterns of two adjacent glomeruli responsible for sex-pheromone processing during postembryonic development of the cockroach Periplaneta americana. Neurosci. Lett. 462 (2009) 219-224.

[14] C.J. Persoons, F.J. Ritter, P.E.J. Verwiet, H. Hauptmann, K. Mori, Nomenclature of American cockroach sex pheromones. Tetrahedron Lett. 31 (1990) 1747-1750. 
[15] L. Prillinger, Postembryonic development of the antennal lobes in Periplaneta americana L. Cell Tissue Res. 215 (1981) 563-575.

[16] W.L. Roelofs, Chemistry of sex attraction. Proc. Natl. Acad. Sci. USA 92 (1995) 44-49.

[17] J.P. Rospars, I. Chambille, Postembryonic growth of the antennal lobe and their identified glomeruli in the cockroach Blaberus craniifer Burm. (Dictyoptera: Blaberidae): A morphometric study. Int, J. Insect Morphol. Embryol. 15 (1986) 393-415.

[18] J.P. Rospars, J.G. Hildebrand, Sexually dimorphic and isomorphic glomeruli in the antennal lobes of the sphinx moth Manduca sexta. Chem. Senses 25 (2000) 119-129.

[19] W. Rössler, L.P. Tolbert, J.G. Hildebrand, Early formation of sexually dimorphic glomeruli in the developing olfactory lobe of the brain of the moth Manduca sexta. J. Comp. Neurol. 396 (1998) 415-428.

[20] R. Schafer, T.V. Sanchez The nature and development of sex attractant specificity in cockroaches of the genus Periplaneta. II. Juvenile hormone regulates sexual dimorphism in the distribution of antennal olfactory receptors. J. Exp. Zool. 198 (1976) 323-336.

[21] D. Schaller, Antennal sensory system of Periplaneta americana L. Distribution and frequency of morphologic types of sensilla and their sex-specific changes during postembryonic development. Cell Tissue Res. 191 (1978) 121-139.

[22] L. Schaller-Selzer, Physiology and morphology of the larval sexual pheromone-sensitive neurons in the olfactory lobe of the cockroach, Periplaneta americana. J. Insect Physiol. 30 (1984) 537-546.

\section{Figure Legends}


Fig. 1. Images of the male macroglomerular complex (MGC) and the female MGC homolog in the right antennal lobe (AL) of the cockroach, viewed anteriorly (a-o), medially $(m, p)$ and dorsally $(n, q)$. (a) Diagram illustrating the positions of the dorsal and ventral antennal nerves and the AL of the adult brain. (b-i) The adult male MGC (b) and the female MGC homolog in larval instars (c-i) are both innervated by the same sensory tract (white arrowhead, b and c) and located in the same location characterized by landmark glomeruli (g1,g3 in b-d,f) and a landmark tract (white arrow, d) projecting to g2 (d). The female MGC homolog is smaller than the male MGC. The A-glomerulus in the female is located more laterally compared to that in the male (b-d). (j-q) A- and B-glomeruli of the adult female in which sensory afferents in the dorsal and ventral antennal nerves are differentially labeled. The images in j) and k) are reconstructed three-dimensionally in l-n and o-q, respectively. Broken lines in $\mathrm{n}$ and $\mathrm{q}$ indicate the border between A- and B-glomeruli. The sensory axons from the dorsal and ventral nerves are almost completely segregated toward the anterior and posterior sides, respectively (black arrows in $\mathrm{m}, \mathrm{q}$ ). Reflecting this axonal segregation, axon terminals from both nerves also tended to be biased toward the anterior and posterior sides $(\mathrm{m}, \mathrm{n}$, p, q). Scale bars $=1 \mathrm{~mm}$ in (a); $100 \mu \mathrm{m}$ in (b, c); $50 \mu \mathrm{m}$ in (d); $20 \mu \mathrm{m}$ in (e-q).

Fig. 2. Log-linear plot of the afferent volumes of A-glomerulus (broken line), B-glomerulus (solid line), and both glomeruli (dotted line) in the female (black) and male (gray) cockroaches during postembryonic development. Each data point is the average value of one antennal lobe from two different animals except for the first instar in which data was obtained from one animal. See supplementary table for values.

Fig. 3. Sexual dimorphism of projection neurons (PNs) in the right brain of the cockroach. (a) The whole projection pattern of PNs. PNs send axons to the calyces of the mushroom body and/or the lateral horn where they terminate (encircled by white solid line). (b-e) Confocal stacks of the anterior region of the lateral horn viewed anteriorly (b,c) and 3D reconstructions of the axon terminals of all PNs in the lateral horn, viewed laterally (d,e), showing the unique projections to the antero-medial region in males, while there are almost absent in females. (f-i) Intracellularly-stained PNs having dendrites in the B-glomerulus (f,h) and one ordinary glomerulus (g,i), showing 
the former has dense terminal arborizations in the antero-medial region of the lateral horn while the terminals of the latter occur in the posterior region. (j-l) The projections to the antero-medial region (encircled by a broken line) are few or absent in larval females (j) and males at the mid-stage of 8th instar males (k) but emerge in late stage of 8th instar males (l). Scale bars $=100 \mu \mathrm{m}$ in (a,d-g); $50 \mu \mathrm{m}$ in (b,c,h-j).

Supplementary table

Table 1. Volumes of afferents in different-aged instars and adult females

\begin{tabular}{cccc}
\hline Age & A & B & A+B \\
\hline 1st & 227 & 810 & 1036 \\
\hline 2nd & 210 & 1435 & 1644 \\
\hline 3rd & 227 & 1644 & 1871 \\
\hline 4th & 575 & 2859 & 3434 \\
\hline 5th & 578 & 4132 & 4710 \\
\hline 6th & 495 & 5019 & 5515 \\
\hline 7th & 1630 & 5411 & 7041 \\
\hline 8th & 1860 & 7691 & 9551 \\
\hline 9th & 2529 & 7871 & 10401 \\
\hline 10th & 4005 & 8878 & 12883 \\
\hline 11th & 3107 & 14019 & 17126 \\
\hline adult & 3805 & 13908 & 17713 \\
\hline
\end{tabular}

Values are means derived from two animals (except for 1st instar in which data is derived from one animal). 


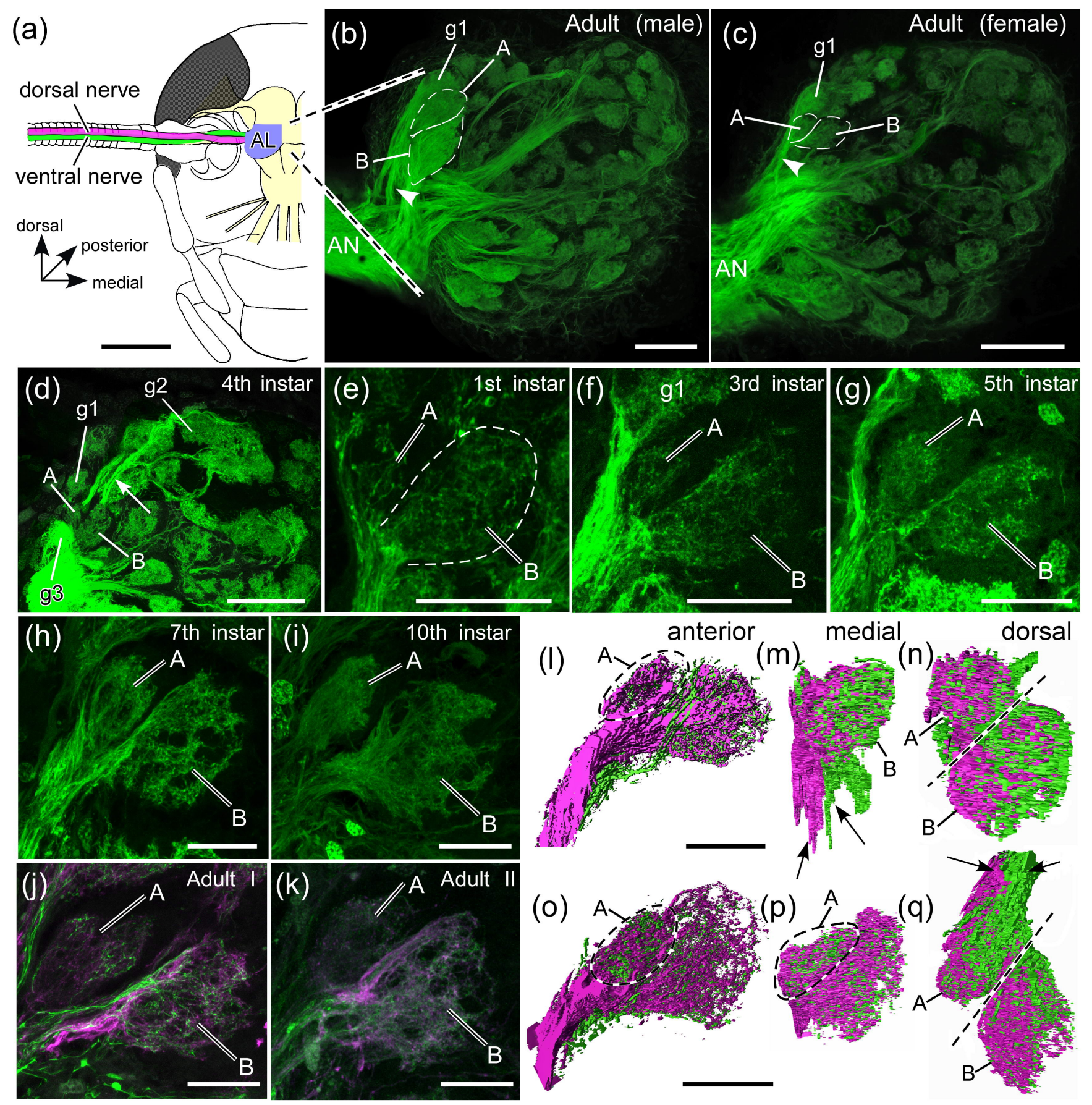


(a) 1000000 - $\mathbf{\Delta}$-female A-glomerulus

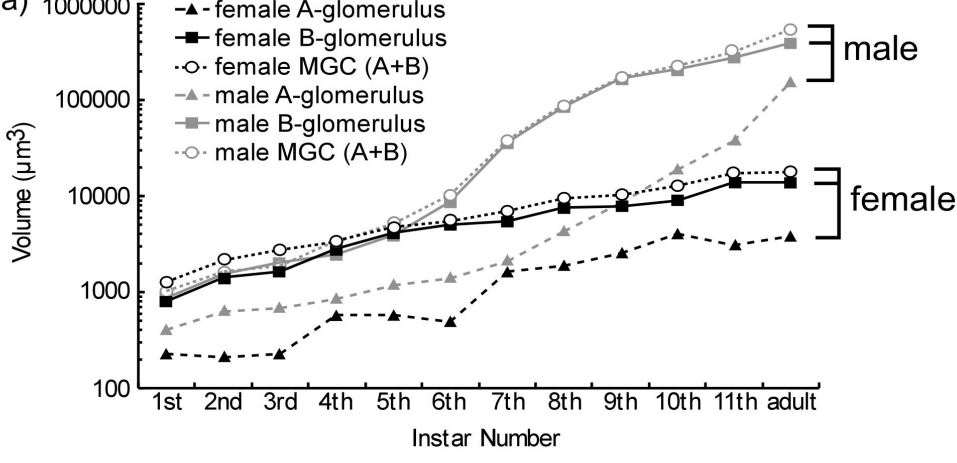




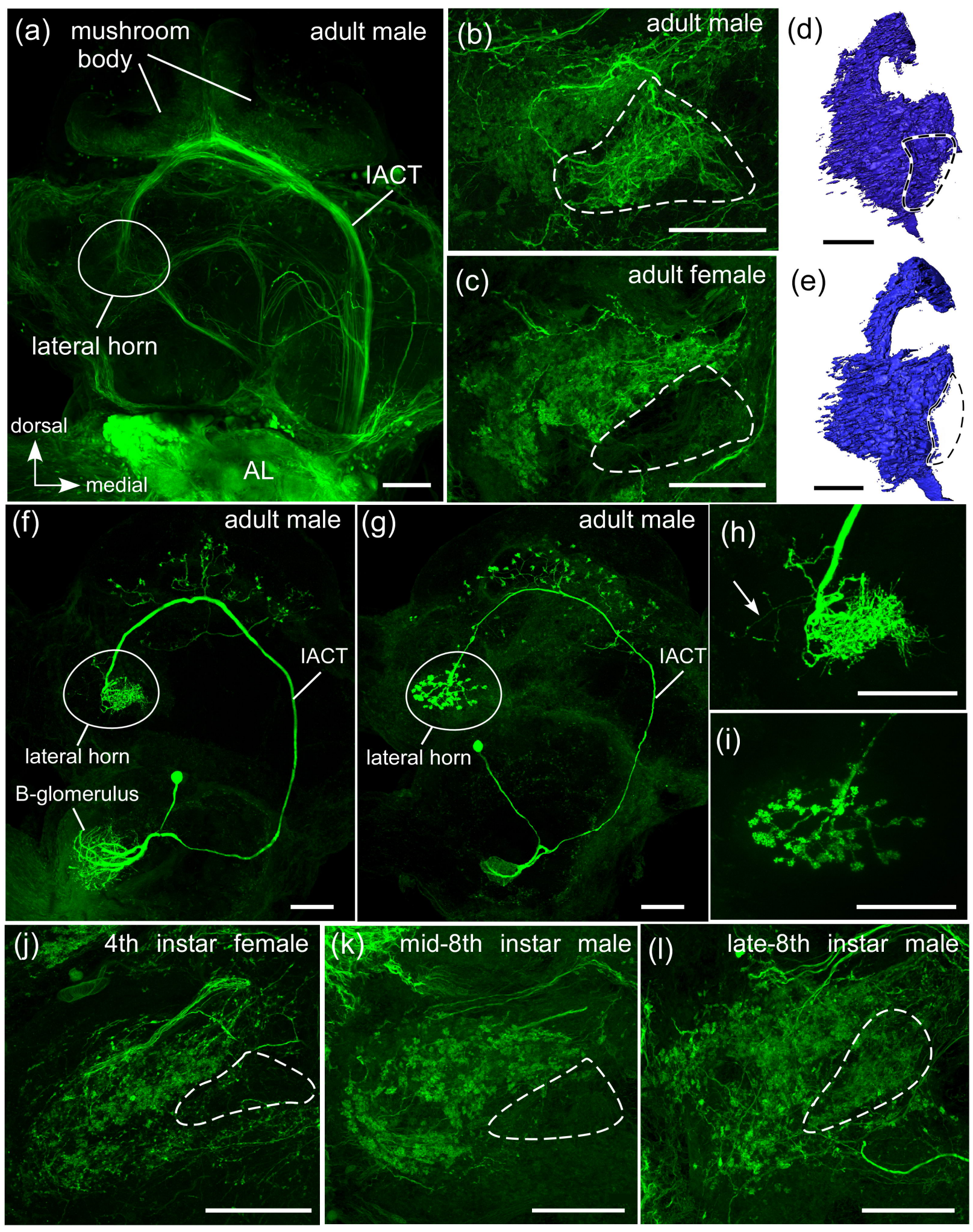

